\title{
Equivariant Hopf Bifurcation in a Time-Delayed Ring of Antigenic Variants
}

\author{
Israel Ncube \\ College of Engineering, Technology, and Physical Sciences, Department of Mathematics, Alabama A \& M University, \\ 4900 Meridian Street North, Huntsville, AL 35762, USA \\ Correspondence should be addressed to Israel Ncube; ncube.israel@gmail.com
}

Received 4 July 2015; Revised 26 November 2015; Accepted 1 December 2015

Academic Editor: Salim Messaoudi

Copyright (C) 2016 Israel Ncube. This is an open access article distributed under the Creative Commons Attribution License, which permits unrestricted use, distribution, and reproduction in any medium, provided the original work is properly cited.

We consider an intrahost malaria model allowing for antigenic variation within a single species. The host's immune response is compartmentalised into reactions to major and minor epitopes. We investigate the dynamics of the model, paying particular attention to bifurcation and stability of the uniform nonzero endemic equilibrium. We establish conditions for the existence of an equivariant Hopf bifurcation in a ring of antigenic variants, characterised by time delay.

\section{Preliminaries}

An intrahost mathematical model of Plasmodium falciparum, a species of parasites that cause malaria in humans, is considered. The central achievement of the model, first proposed by [1], is its ability to replicate the phenomenon of antigenic variation, which is a mechanism employed by the parasite in order to evade detection by the host's immune system. In addition, the proposed model incorporates the effects of immune response (IR) mounted by the human host. Such a model has been the subject of a number of previous studies (see [2-11], e.g.). In particular, $[7,8]$ introduce the idea of a delayed IR, leading to a mathematical model comprised of a set of coupled nonlinear delay differential equations (DDEs), where it is assumed that the IR time delay is discrete. Specifically, there is a time delay between changes in the parasite load and the production of immune effectors [8]. The authors then proceed to show that a range of interesting dynamics (synchronous and asynchronous oscillations) result as a consequence of the (small) time delay. The current paper is a further development of the model studied in $[7,8]$. The distinction of our work is that we focus on the effects of symmetry on the dynamics of the model and that our time delay is not constrained to be small. In particular, we establish sufficient and necessary conditions for the existence of an equivariant Hopf bifurcation. We must state at the onset that the current study is similar in spirit to the recent work of [3-5]. However, there are fundamental differences in what we do with the model, as explained below. First of all, the study conducted in [3] concerns the Recker et al. [1] model, with no time delays in the host's immune response. Following in the footsteps of the monumental work of $[12,13]$, the author then uses elements of equivariant bifurcation theory to study the effects of symmetry on the dynamic interactions of the host and the pathogens. In the work of [4], the authors attack the problem of symmetry-breaking in system (1). They establish the existence of a fully symmetric steady state of (1) and then employ ideas of equivariant bifurcation theory $[12,13]$ to study the dynamics of this steady state. Essentially, the authors investigate the effects of immune response time delay on the symmetric dynamics of (1). They do so by employing the technique of isotypic decomposition [12, 13] to reduce the stability problem to a simple transcendental equation for the eigenvalues [4]. In [5], the authors employ the groupoid formalism developed in $[14,15]$ to study the dynamics of cross-reactivity from antigenic variation and establish a synchrony-breaking Hopf bifurcation emanating from a nontrivial synchronous equilibrium of system (1). To 
the best of our knowledge, the problem of equivariant Hopf bifurcation in the time-delayed modification of the Recker et al. [1] model (2) or (1) has never been addressed before in the literature.

Let us begin by commenting that an in-depth description of time-delayed modification of Recker et al. [1] model can be found in $[7,8]$. Here we simply give a very brief description, primarily for the express purpose of casting the model in the context of the analysis to come. The timedelayed modification of Recker et al. [1] is expressible in the form $[7,8]$

$$
\begin{aligned}
Y_{j}^{\prime}(T) & =\phi Y_{j}(T)-\alpha Z_{j}(T) Y_{j}(T)-\alpha^{\prime} W_{j}(T) Y_{j}(T), \\
Z_{j}^{\prime}(T) & =\beta Y_{j}\left(T-T_{d}\right)-\mu Z_{j}(T), \\
W_{j}^{\prime}(T) & =\beta^{\prime} \sum_{k=1}^{N} c_{j k} Y_{k}\left(T-T_{d}\right)-\mu^{\prime} W_{j}(T),
\end{aligned}
$$

where the index $j=1, \ldots, N$ separates the parasitised red blood cell population, denoted by $Y_{j}$, into $N$ variants, each characterised by the unique major epitope of their displayed antigen (see $[1,7,8]$ and references cited therein). The variables $Z_{j}$ and $W_{j}$ denote variant-specific and crossreactive immune responses, respectively; $\phi$ is the intrinsic parasite growth rate, $\alpha$ and $\alpha^{\prime}$ are the removal rates associated with specific and cross-reactive immune responses, respectively, $\beta$ and $\beta^{\prime}$ are the proliferation rates of immune responses, $\mu$ and $\mu^{\prime}$ are the decay rates of variant-specific and cross-reactive immune responses, and $T_{d}$ is the discrete time delay of the IR. The coefficients $c_{j k}$ of the connectivity matrix characterise cross-reactive intervariant interactions $[1,2,7,8,11]$.

After normalisation and change of variables, [8] reduced system (1) to the following system:

$$
\begin{aligned}
y_{j}^{\prime}(t)= & -x_{j}(t)\left[1+y_{j}(t)\right] \\
x_{j}^{\prime}(t)= & \frac{c}{n} y_{j}(t-\tau)+\frac{1}{n} \sum_{k=1}^{n} y_{k}(t-\tau)-a x_{j}(t) \\
& +a(1-b) w_{j}(t) \\
w_{j}^{\prime}(t)= & \frac{1}{n} \sum_{k=1}^{n} y_{k}(t-\tau)-a b w_{j}(t),
\end{aligned}
$$

where $\tau \in \mathbb{R}^{+}$is a discrete time delay. The index $j=$ $1, \ldots, n<N$ separates the parasitised red blood cell population, denoted by $y_{j}$, into $n<N$ variants. The variants $j=n+1, \ldots, N$ are neglected in this reformulation of (1) [8]. As a consequence of this, the sum in (1) collapses to [8]

$$
\sum_{k=1}^{N} c_{j k} Y_{k}\left(T-T_{d}\right)=\sum_{k=1}^{n} Y_{k}\left(T-T_{d}\right)
$$

Without going into specific details, it is important to point out that all the parameters in (2) are positive. Every variant in system (2) has the same $n$ minor epitopes in common [7]. This point highlights a fundamental difference between the model studied in this work and the models studied in [35]. In essence, (2) represents a subsystem of (1). System (2) represents the interaction of malaria antigenic variants in the special case in which there are $n$ minor epitopes characterised by $n$ variants per epitope. The total number of variants in this case is given by

$$
\prod_{j=1}^{n} n=n^{n}
$$

The interaction of these different antigenic variants may be represented schematically as shown in Figure 1, from which it is evident that system (2) is endowed with some spatial symmetry, which we will attempt to describe in due course. We may gain some further insight about system (2) by analysing the structure of its associated adjacency matrix $\mathbf{T}$, whose entries $T_{i j}$ are identical to unity if the variants $i$ and $j$ have some minor epitopes in common; otherwise $T_{i j}=0$ [4]. The matrix $\mathbf{T}$ is always symmetric [4]. In the case of a ring of $n^{n}$ variants characterised by all-to-all coupling, as depicted in Figure 1, the corresponding $n^{n} \times n^{n}$ adjacency matrix is given by

$$
\mathbf{T}=\left(\begin{array}{cccccccccc}
1 & 1 & 1 & 1 & 1 & 1 & \cdots & 1 & 1 & 1 \\
1 & 1 & 1 & 1 & 1 & 1 & \cdots & 1 & 1 & 1 \\
1 & 1 & 1 & 1 & 1 & 1 & \cdots & 1 & 1 & 1 \\
1 & 1 & 1 & 1 & 1 & 1 & \cdots & 1 & 1 & 1 \\
\vdots & \vdots & \vdots & \vdots & \vdots & \vdots & \cdots & \vdots & \vdots & \vdots \\
1 & 1 & 1 & 1 & 1 & 1 & \cdots & 1 & 1 & 1
\end{array}\right)
$$

It is straightforward to construct the adjacency matrix $\mathbf{T}$ for an arbitrarily large number of minor epitopes [3]. In this paper, we focus on $n$ minor epitopes, with $n$ antigenic variants per epitope. By recourse to (5), we may express (2) in vectorial form as

$$
\begin{aligned}
& \frac{d}{d t}\left(\begin{array}{c}
\mathbf{y}(t) \\
\mathbf{x}(t) \\
\mathbf{w}(t)
\end{array}\right) \\
& =\left\{\begin{array}{l}
-\mathbf{x}(t)\left[\mathbf{1}_{n}+\mathbf{y}(t)\right], \\
\frac{c}{n} \mathbf{y}(t-\tau)+\frac{1}{n} \mathbf{T y}(t-\tau)-a \mathbf{x}(t)+a(1-b) \mathbf{w}(t), \\
\frac{1}{n} \mathbf{T y}(t-\tau)-a b \mathbf{w}(t),
\end{array}\right.
\end{aligned}
$$

where $\mathbf{y}=\left(y_{1}, y_{2}, \ldots, y_{n}\right)^{\mathrm{T}}, \mathbf{x}=\left(x_{1}, x_{2}, \ldots, x_{n}\right)^{\mathrm{T}}, \mathbf{w}=$ $\left(w_{1}, w_{2}, \ldots, w_{n}\right)^{\mathrm{T}}$, and $\mathbf{1}_{n}=(\overbrace{1,1, \ldots, 1}^{n \text { times }})^{\mathrm{T}}$. With appropriate initial conditions, it may be easily shown that system (6) is well posed [4]; that is, its solutions are nonnegative $\forall t \geq 0$. Symmetry properties of (6) are encoded in the associated 


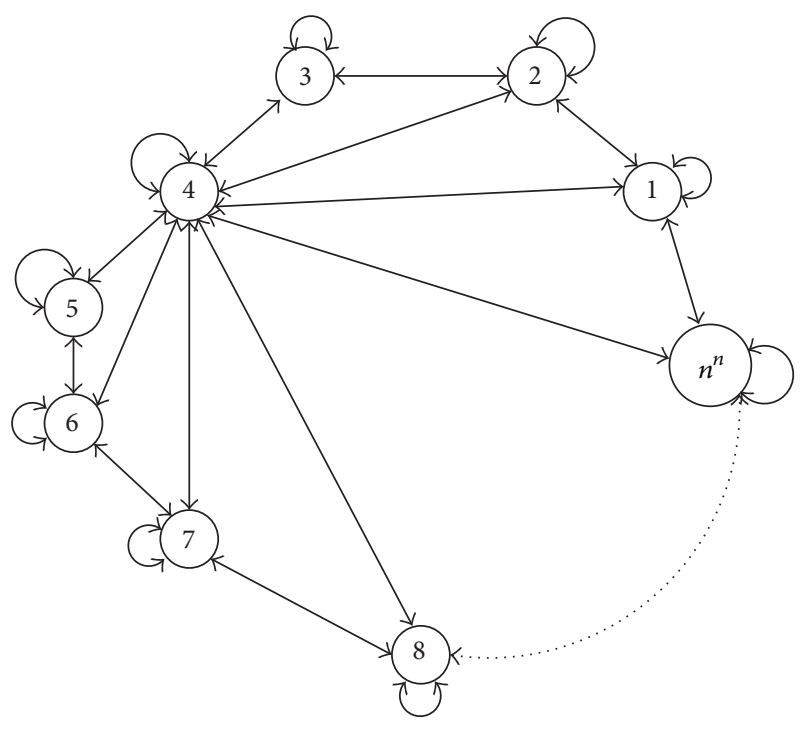

FIGURE 1: Interaction of $n^{n}$ antigenic variants in the case of $n$ minor epitopes with $n$ variants per epitope. Every variant in the ring will be connected to every other variant in much the same way that variant 4 is connected. For clarity and to avoid cluttering the diagram, we have only shown the full network connections of variant 4 .

adjacency matrix $\mathbf{T}$. In the present case, in which there are $n$ minor epitopes with $n$ variants per epitope, the dynamical system (6) is equivariant with respect to the symmetry group $[12,13]$

$$
\Gamma:=\underbrace{\mathbb{S}_{n} \times \mathbb{S}_{n} \times \mathbb{S}_{n} \times \cdots \times \mathbb{S}_{n}}_{n \text { times }} \times \mathbb{Z}_{n}
$$

where $\mathbb{S}_{n}$ represents the symmetric group of all permutations in a network of $n$ nodes with an all-to-all coupling and $\mathbb{Z}_{n}$ is the cyclic group of order $n$, corresponding to rotations by $2 \pi / n[4,12,13]$. In particular, system (6) is equivariant under the action of the dihedral group $\mathbb{D}_{n^{n}}$, which is a $2 n^{n}$ dimensional symmetry group of an $n^{n}$-gon (see [3] for a pertinent brief outline of equivariance bifurcation theory). For the general dihedral group $\mathbb{D}_{n^{n}}$ of order $2 n^{n}$, whether $n^{n}$ is even or odd is crucial as it demarcates two different choices as far as conjugacies of reflections are concerned (see page 128 of [3]).

System (2) has an infinitude of $3 n$-dimensional equilibria, of the generic form

E

$$
:=(\underbrace{y_{1}^{*}, y_{2}^{*}, \ldots, y_{n}^{*}}_{n \text { times }}, \underbrace{x_{1}^{*}, x_{2}^{*}, \ldots, x_{n}^{*}}_{n \text { times }}, \underbrace{w_{1}^{*}, w_{2}^{*}, \ldots, w_{n}^{*}}_{n \text { times }}),
$$

where

$$
\begin{aligned}
& x_{j}^{*}=0, \\
& y_{j}^{*}=-\frac{a n}{c} w_{j}^{*},
\end{aligned}
$$

$$
\begin{aligned}
w_{j}^{*}=\frac{1}{a b n} \sum_{k=1}^{n} y_{k}^{*}, & \\
& j=1,2, \ldots, n .
\end{aligned}
$$

We must comment at this point that the present study focusses entirely on the uniform equilibrium

$$
E_{0}:=(\underbrace{0, \ldots, 0}_{n \text { times }}, \underbrace{0, \ldots, 0}_{n \text { times }}, \underbrace{0, \ldots, 0}_{n \text { times }})
$$

of (2). The linearisation of system (2) about the equilibrium $E_{0}$ is given by

$$
\begin{aligned}
y_{j}^{\prime}(t)= & -x_{j}(t), \\
x_{j}^{\prime}(t)= & \frac{c}{n} y_{j}(t-\tau)+\frac{1}{n} \sum_{k=1}^{n} y_{k}(t-\tau)-a x_{j}(t) \\
& +a(1-b) w_{j}(t), \\
w_{j}^{\prime}(t)= & \frac{1}{n} \sum_{k=1}^{n} y_{k}(t-\tau)-a b w_{j}(t)
\end{aligned}
$$

and yields the characteristic equation [7]

$$
D(\lambda, \tau):=\left(F_{1}(\lambda) F_{a p}(\lambda, \tau)\right)^{n-1} F_{s}(\lambda, \tau)=0
$$

where

$$
\begin{aligned}
F_{1}(\lambda):= & \lambda+a b, \\
F_{a p}(\lambda, \tau):= & \lambda^{2}+a \lambda+\frac{c}{n} e^{-\lambda \tau}, \\
F_{s}(\lambda, \tau):= & \lambda^{3}+a(1+b) \lambda^{2}+a^{2} b \lambda \\
& +e^{-\lambda \tau}\left[\lambda\left(1+\frac{c}{n}\right)+a\left(1+\frac{b c}{n}\right)\right]
\end{aligned}
$$

and where all the parameters are nonnegative. Equation (12) consists of the factors $\Delta_{1}(\lambda, \tau):=F_{1}(\lambda) F_{a p}(\lambda, \tau)$ and $\Delta_{2}(\lambda, \tau):=F_{s}(\lambda, \tau)$, with multiplicities $n-1$ and 1 , respectively. This type of factorisation of the characteristic equation is due to the presence of symmetry in (2). The continuous extension at $\lambda=0$ is given by

$$
D(0, \tau)=\lim _{\lambda \rightarrow 0} D(\lambda, \tau)=a^{n}\left(\frac{b c}{n}\right)^{n-1} \cdot\left(1+\frac{b c}{n}\right)>0 .
$$

The equilibrium $E_{0}$ of (2) will be locally asymptotically stable if all of the roots, $\lambda$, of (12) have negative real part and unstable if at least one root has a positive real part. 
First of all, we must note that $F_{1}(\lambda)=0$ if, and only if, $\lambda=$ $-a b<0$. In other words, $F_{1}(\lambda)=0$ always yields a real root of (12), $\lambda=-a b<0$, of multiplicity $n-1$. As a consequence of this observation, we see that an analysis of the distribution of roots of $\Delta_{1}(\lambda, \tau)=0$ boils down to that of the distribution of roots of the factor $F_{a p}(\lambda, \tau)=0$. Let $\lambda=\nu+i \omega, \nu, \omega \in \mathbb{R}$ in the two factors, $F_{a p}(\lambda, \tau)$ and $\Delta_{2}(\lambda, \tau)$, of (12), and separate into real and imaginary parts to obtain $\Delta_{j}(\lambda, \tau)=R_{j}(\nu, \omega)+$ $i I_{j}(\nu, \omega)$, with $j=1,2$, where

$$
\begin{aligned}
& R_{1}(\nu, \omega)=a \nu+\nu^{2}-\omega^{2}+\frac{c}{n} e^{-\tau \nu} \cos (\omega \tau) \\
& I_{1}(\nu, \omega)=a \omega+2 \nu \omega-\frac{c}{n} e^{-\tau \nu} \sin (\omega \tau) \\
& R_{2}(\nu, \omega) \\
& =v\left(\nu^{2}-\omega^{2}\right)-2 v \omega^{2}+a(1+b)\left(\nu^{2}-\omega^{2}\right)+a^{2} b \nu \\
& \quad+e^{-\nu \tau}\left[\nu\left(1+\frac{c}{n}\right)+a\left(1+\frac{b c}{n}\right)\right] \cos (\omega \tau) \\
& \quad-e^{-\nu \tau} \omega\left(1+\frac{c}{n}\right) \sin (\omega \tau), \\
& I_{2}(\nu, \omega) \\
& =\omega\left(\nu^{2}-\omega^{2}\right)+2 \nu^{2} \omega+2 a(1+b) \nu \omega+\omega a^{2} b
\end{aligned}
$$

$$
\begin{aligned}
& \sin (\omega \tau)=\frac{a n \omega}{c}, \\
& \cos (\omega \tau)=\frac{n \omega^{2}}{c}, \\
& \sin (\omega \tau)=\frac{n\left[\omega^{3}-\omega a^{2} b-a(1+b) \omega^{2}\right]}{\omega(n+c)-a(n+b c)}, \\
& \cos (\omega \tau)=\frac{n\left(a(1+b) \omega^{2}[\omega(n+c)-a(n+b c)]+\omega(n+c)\left[\omega^{3}-\omega a^{2} b-a(1+b) \omega^{2}\right]\right)}{a[\omega(n+c)-a(n+b c)](n+b c)},
\end{aligned}
$$

$$
\begin{aligned}
& +e^{-\nu \tau} \omega\left(1+\frac{c}{n}\right) \cos (\omega \tau) \\
& -e^{-\nu \tau}\left[\nu\left(1+\frac{c}{n}\right)+a\left(1+\frac{b c}{n}\right)\right] \sin (\omega \tau) .
\end{aligned}
$$

We begin our investigation by focussing on the factor $\Delta_{1}(\lambda, \tau)$ of (12). It is of vital importance to establish conditions under which the factor $F_{a p}(\lambda, \tau)$ has purely imaginary roots. To this end, let $v=0$ in (15). This leads to the following simplifying relations:

$$
\begin{aligned}
& -n \omega^{2}+c \cos (\omega \tau)=0, \\
& n a \omega-c \sin (\omega \tau)=0, \\
& a\left(1+\frac{b c}{n}\right) \cos (\omega \tau)-\omega\left(1+\frac{c}{n}\right) \sin (\omega \tau) \\
& \quad=a(1+b) \omega^{2}, \\
& a\left(1+\frac{b c}{n}\right) \sin (\omega \tau)-\omega\left(1+\frac{c}{n}\right) \cos (\omega \tau) \\
& \quad=-\omega^{3}+\omega a^{2} b,
\end{aligned}
$$

respectively. Solving for $\sin (\omega \tau)$ and $\cos (\omega \tau)$ in (16) and (17) yields respectively. Recalling that $\sin ^{2}(\omega \tau)+\cos ^{2}(\omega \tau)=1$, squaring both sides of the equations in (18), adding, and rearranging give

$$
\Phi(\omega):=n^{2} \omega^{4}+a^{2} n^{2} \omega^{2}-c^{2}
$$

which always has at least one real root $\omega_{*}>0$, since $\Phi(0)=$ $-c^{2}<0$. In fact, the possible solutions of the quartic $\Phi(\omega)=0$ are given by

$$
\begin{aligned}
& \omega_{+}=\frac{\sqrt{n\left(-a^{2} n+\sqrt{\left.a^{4} n^{2}+4 c^{2}\right)}\right.}}{n \sqrt{2}}>0, \\
& \omega_{-}=-\frac{\sqrt{n\left(-a^{2} n+\sqrt{a^{4} n^{2}+4 c^{2}}\right)}}{n \sqrt{2}}<0 .
\end{aligned}
$$

Since the frequency $\omega>0$, it follows that the only viable solution of $(20)$ is $\omega_{+}$. This implies that the characteristic equation (12) has $(n-1)$ pairs of purely imaginary roots $\pm i \omega_{+}$. Similarly, (19) yields the octic polynomial

$$
\begin{aligned}
\Gamma(\omega):= & A_{8} \omega^{8}+A_{6} \omega^{6}+A_{5} \omega^{5}+A_{4} \omega^{4}+A_{3} \omega^{3} \\
& +A_{2} \omega^{2}+A_{1} \omega+A_{0},
\end{aligned}
$$

where the coefficients $A_{0}, \ldots, A_{8}$ are given in the Appendix. We now quickly review the local stability of $E_{0}$. We focus our attention on the factor $\Delta_{1}(\lambda, \tau)$ of (12). Equation (20) has one real root, denoted by $\omega_{+}$. Substitute $\omega_{+}$into (18) to obtain the sequence of critical time delays

$$
\tau_{+}^{(j)}=\frac{1}{\omega_{+}} \cos ^{-1}\left(\frac{n \omega_{+}^{2}}{c}\right)+\frac{2 \pi j}{\omega_{+}}, \quad j=0,1,2,3, \ldots
$$


As a result, when $\tau=\tau_{+}^{(j)}$, the characteristic equation (12) has $(n-1)$ pairs of purely imaginary roots; $\lambda= \pm i \omega_{+}$. We define

$$
\tau_{01}:=\min \left\{\tau_{+}^{(j)} \mid j=0,1,2,3, \ldots\right\} .
$$

Let us shift our attention momentarily to the factor $\Delta_{2}(\lambda, \tau)$ of (12). First of all, we note that (22) has at least one positive real root if $A_{0}<0$ and $A_{8}>0$. The proof of this fact is indeed elementary. Assume that (22) has eight simple real roots, denoted by $\omega_{k}, k=1,2, \ldots, 8$. In fact, establishing conditions to guarantee the existence of such roots of (22) is far from trivial. For this reason, we will avoid delving into this subject in this paper. Suffice to say that the existence of such roots guarantees that a nondegenerate bifurcation occurs in the special case $A_{0}>0, A_{8}>0$. To establish that one of these roots is positive, we proceed in the following manner. Assume that $A_{8} \neq 0$, and define the function

$$
\begin{aligned}
\bar{\Gamma}(\omega)= & \omega^{8}+\bar{A}_{6} \omega^{6}+\bar{A}_{5} \omega^{5}+\bar{A}_{4} \omega^{4}+\bar{A}_{3} \omega^{3}+\bar{A}_{2} \omega^{2} \\
& +\bar{A}_{1} \omega+\bar{A}_{0},
\end{aligned}
$$

where $\bar{A}_{6}:=A_{6} / A_{8}, \bar{A}_{5}:=A_{5} / A_{8}, \bar{A}_{4}:=A_{4} / A_{8}, \bar{A}_{3}:=$ $A_{3} / A_{8}, \bar{A}_{2}:=A_{2} / A_{8}, \bar{A}_{1}:=A_{1} / A_{8}$, and $\bar{A}_{0}:=A_{0} / A_{8}$. It is important to stress that one of the roots of (22) is positive if at least one of the critical points of (25) is positive. The derivative function of (25) is

$$
\begin{gathered}
\bar{\Gamma}^{\prime}(\omega)=8\left(\omega^{7}+\frac{3}{4} \bar{A}_{6} \omega^{5}+\frac{5}{6} \bar{A}_{5} \omega^{4}+\frac{1}{2} \bar{A}_{4} \omega^{3}\right. \\
\left.+\frac{3}{8} \bar{A}_{3} \omega^{2}+\frac{1}{4} \bar{A}_{2} \omega+\frac{1}{8} \bar{A}_{1}\right) .
\end{gathered}
$$

Function (26) has at least one positive real root if, and only if, at least one of its critical points is positive. This happens if, and only if, $A_{2}<0$. Consequently, one of the roots of (22) is positive if, and only if, the condition $A_{2}<0$ is fulfilled. From (19), we compute the corresponding sequence of critical time delays:

$$
\tau_{k}^{(j)}=\frac{1}{\omega_{k}} \cos ^{-1}\left(\frac{n\left(a(1+b) \omega_{k}^{2}\left[\omega_{k}(n+c)-a(n+b c)\right]+\omega_{k}(n+c)\left[\omega_{k}^{3}-\omega_{k} a^{2} b-a(1+b) \omega_{k}^{2}\right]\right)}{a\left[\omega_{k}(n+c)-a(n+b c)\right](n+b c)}\right)+\frac{2 \pi j}{\omega_{k}}
$$

where $j=0,1,2, \ldots$ and $k=1,2, \ldots, 8$. When $\tau=\tau_{k}^{(j)}$, it follows that (12) has a pair of purely imaginary roots, $\lambda=$ $\pm i \omega_{k}$. Finally, we define

$$
\tau_{02}:=\min \left\{\tau_{k}^{(j)} \mid k=1,2, \ldots, 8 ; j=0,1,2,3, \ldots\right\} .
$$

Assume that $\lambda=\lambda(\tau)$ in (12), and differentiate with respect to $\tau$. For the simple root case, we recall from (12) that $\Delta_{2}(i \omega, \tau)=$ 0 . As a consequence of this, we obtain the following:

$$
\operatorname{Re}\left(\left.\frac{d \lambda}{d \tau}\right|_{\lambda=i \omega}\right)=\frac{(a+a b c / n) \omega \sin (\omega \tau)-\omega(\omega+\omega c / n) \cos (\omega \tau)+i[\omega(a+a b c / n) \cos (\omega \tau)+\omega(\omega+\omega c / n) \sin (\omega \tau)]}{\xi_{1}+i \xi_{2}}
$$

where

$$
\begin{aligned}
\xi_{1}= & a^{2} b-3 \omega^{2}+\left(1+\frac{c}{n}\right) \cos (\omega \tau) \\
& -\tau\left(a+\frac{a b c}{n}\right) \cos (\omega \tau) \\
& -\tau\left(\omega+\frac{\omega c}{n}\right) \sin (\omega \tau), \\
\xi_{2}= & 2 a \omega b+2 a \omega-\left(1+\frac{c}{n}\right) \sin (\omega \tau) \\
& +\tau\left(a+\frac{a b c}{n}\right) \sin (\omega \tau) \\
& -\tau\left(\omega+\frac{\omega c}{n}\right) \cos (\omega \tau) .
\end{aligned}
$$

Using the computer algebra package MAPLE ${ }^{\circledast}$, we deduce that the usual transversality condition corresponding to the factor $\Delta_{2}(\lambda, \tau)$ is fulfilled if, and only if,

$$
\begin{gathered}
-\omega_{k} c a^{2} b n \cos \left(\omega_{k} \tau_{k}^{(j)}\right)-2 \omega_{k} a^{2} b^{2} c n \cos \left(\omega_{k} \tau_{k}^{(j)}\right) \\
+\omega_{k}^{2} a b c n \sin \left(\omega_{k} \tau_{k}^{(j)}\right)-a^{3} b^{2} c n \sin \left(\omega_{k} \tau_{k}^{(j)}\right) \\
-2 \omega_{k}^{2} \operatorname{can} \sin \left(\omega_{k} \tau_{k}^{(j)}\right)-\omega_{k} a^{2} b n^{2} \cos \left(\omega_{k} \tau_{k}^{(j)}\right) \\
-2 \omega_{k}^{2} a b n^{2} \sin \left(\omega_{k} \tau_{k}^{(j)}\right)+2 \omega_{k} c n \\
-3 \omega_{k}^{3} n^{2} \cos \left(\omega_{k} \tau_{k}^{(j)}\right)+\omega_{k}\left(c^{2}+n^{2}\right) \\
-3 c n \omega_{k}^{3} \cos \left(\omega_{k} \tau_{k}^{(j)}\right)-a^{3} b n^{2} \sin \left(\omega_{k} \tau_{k}^{(j)}\right)
\end{gathered}
$$




$$
\begin{aligned}
& +\omega_{k}^{2} a n^{2} \sin \left(\omega_{k} \tau_{k}^{(j)}\right)-2 \omega_{k} a^{2} n^{2} \cos \left(\omega_{k} \tau_{k}^{(j)}\right) \\
& \neq 0 .
\end{aligned}
$$

By continuity, it follows that $\operatorname{Re}[\lambda(\tau)]$ becomes positive when $\tau>\tau_{k}^{(0)}$ and the equilibrium $E_{0}$ of (2) becomes unstable. As a result, a simple root Hopf bifurcation occurs when $\tau$ passes through the critical time delay $\tau_{k}^{(0)}$. Consider the equation $\Delta_{1}(\lambda, \tau)=0$ when $\tau=0$; that is,

$$
\begin{aligned}
\Delta_{1}(\lambda, 0) & :=\lambda^{3}+a(1+b) \lambda^{2}+\left(\frac{c}{n}+a^{2} b\right) \lambda+\frac{a b c}{n} \\
& =0 .
\end{aligned}
$$

Employing the well-known Routh-Hurwitz criterion and the fact that the parameters $a, b, c$, and $n$ are strictly positive, it follows that all the roots of (32) have negative real part if, and only if,

$$
a\left(\frac{c}{n}+a^{2} b\right)(1+b)-\frac{a b c}{n}>0 .
$$

Similarly, for the equation $\Delta_{2}(\lambda, \tau)=0$ with $\tau=0$, the RouthHurwitz criterion implies that all the roots of this equation have negative real part if, and only if,

$$
\left[a\left(\frac{c}{n}+a^{2} b\right)(1+b)-\frac{a b c}{n}\right]+a b>0 .
$$

Proposition 1. For $\tau=0$, the equilibrium $E_{0}$ of (2) is asymptotically stable if, and only if, the inequality in (33) is fulfilled.

We arrive at the first of our main results.

Theorem 2. Suppose that conditions (34) and (31) are satisfied and that (20) has no positive real roots for $\tau \in(0, \widetilde{\tau}]$, where $\widetilde{\tau}>$ $\tau_{k}^{(0)}$. Since $\Phi(0)=-c^{2}<0$, it follows that $E_{0}$ is asymptotically stable when $\tau<\tau_{k}^{(0)}$ and unstable when $\tau_{k}^{(0)}<\tau<\min \left\{\tilde{\tau}, \tau_{k}^{(1)}\right\}$. When $\tau=\tau_{k}^{(0)}$, a simple root Hopf bifurcation occurs.

The characteristic equation (12) can be written in the form

$$
D(\lambda, \tau)=\Delta_{1}^{n-1}(\lambda, \tau) \Delta_{2}(\lambda, \tau) .
$$

By inspection, we can see that (35) has purely imaginary roots $\lambda= \pm i \omega$ of multiplicity $n-1$ for parameters such that $\Delta_{1}( \pm i \omega, \tau)=0$. That is, when

$$
\begin{aligned}
& a b\left(-\omega^{2}+\frac{c}{n} \cos (\omega \tau)\right)-\omega\left(a \omega-\frac{c}{n} \sin (\omega \tau)\right)=0, \\
& a b\left(a \omega-\frac{c}{n} \sin (\omega \tau)\right)-\omega\left(-\omega^{2}+\frac{c}{n} \cos (\omega \tau)\right)=0 .
\end{aligned}
$$

The set of equations of (16) has four parameters, namely, $(a, \tau, c, n)$. If we fix three of the parameters, this gives two equations that may be solved for the critical value of the fourth parameter and the corresponding imaginary part of the eigenvalue $\omega_{c}$. We consider $a$ as the bifurcation parameter. All the results hold and are proved analogously, if any of the other parameters is used instead [16]. For convenience, we rewrite (16) in the form

$$
\begin{aligned}
& c \cdot \sin (\omega \tau)=n a \omega, \\
& c \cdot \cos (\omega \tau)=n \omega^{2} .
\end{aligned}
$$

Taking the ratio of the two expressions in (37) yields an implicit equation for $\omega_{c}$ :

$$
\omega_{c} \tan \left(\omega_{c} \tau\right)-a=0
$$

Squaring and adding the expressions of (37) yield an equation for the corresponding critical value of $a$ for $\Delta_{1}$ :

$$
n^{2} \omega^{4}+n^{2} a^{2} \omega^{2}-c^{2}=0 .
$$

Solving (39) for the parameter $a$ gives

$$
a_{c}\left(\omega_{c}\right)= \begin{cases}\tilde{a}_{+}\left(\omega_{c}\right), & \text { if } \frac{c}{n \omega_{c}} \sin \left(\omega_{c} \tau\right)>0, \\ \tilde{a}_{-}\left(\omega_{c}\right), & \text { if } \frac{c}{n \omega_{c}} \sin \left(\omega_{c} \tau\right)<0,\end{cases}
$$

where

$$
\begin{aligned}
& \tilde{a}_{+}\left(\omega_{c}\right)=\sqrt{\frac{c^{2}-n^{2} \omega_{c}^{4}}{n^{2} \omega_{c}^{2}},} \\
& \tilde{a}_{-}\left(\omega_{c}\right)=-\sqrt{\frac{c^{2}-n^{2} \omega_{c}^{4}}{n^{2} \omega_{c}^{2}}} .
\end{aligned}
$$

Let $\mathscr{C}:=C\left([-\tau, 0], \mathbb{R}^{3 n}\right)$ denote the Banach space of continuous mappings from $[-\tau, 0]$ into $\mathbb{R}^{3 n}$ and endowed with the norm

$$
\|\phi\|_{\tau}=\sup _{-\tau \leq \theta \leq 0}|\phi(\theta)|, \quad \text { for } \phi \in \mathscr{C}
$$

where $|\cdot|$ is the Euclidean norm on $\mathbb{R}^{3 n}$. Let $\mathbf{x}(t)$ be a solution of (2), and define $\mathbf{x}_{t}(\theta)=\mathbf{x}(t+\theta),-\tau \leq \theta \leq 0$. If the solution $\mathbf{x}(t)$ is continuous, then $\mathbf{x}_{t}(\theta) \in \mathscr{C}$. We may now express (2) as the functional differential equation

$$
\mathbf{x}^{\prime}(t)=\mathbf{F}\left(\mathbf{x}_{t}\right),
$$

where $\mathbf{F}: \mathscr{C} \rightarrow \mathbb{R}^{3 n}$ is defined by 


$$
\left.\mathbf{F}(\phi):=\left(\begin{array}{c}
-\phi_{21}(0)\left[1+\phi_{11}(0)\right] \\
-\phi_{22}(0)\left[1+\phi_{12}(0)\right] \\
\vdots \\
-\phi_{2 n}(0)\left[1+\phi_{1 n}(0)\right] \\
\frac{c}{n} \phi_{11}(-\tau)+\frac{1}{n} \sum_{k=1}^{n} \phi_{1 k}(-\tau)-a \phi_{21}(0)+a(1-b) \phi_{31}(0) \\
\frac{c}{n} \phi_{12}(-\tau)+\frac{1}{n} \sum_{k=1}^{n} \phi_{1 k}(-\tau)-a \phi_{22}(0)+a(1-b) \phi_{32}(0) \\
\vdots \\
\frac{c}{n} \phi_{1 n}(-\tau)+\frac{1}{n} \sum_{k=1}^{n} \phi_{1 k}(-\tau)-a \phi_{2 n}(0)+a(1-b) \phi_{3 n}(0) \\
\frac{1}{n} \sum_{k=1}^{n} \phi_{1 k}(-\tau)-a b \phi_{31}(0) \\
\frac{1}{n} \sum_{k=1}^{n} \phi_{1 k}(-\tau)-a b \phi_{32}(0) \\
\vdots \\
\frac{1}{n} \sum_{k=1}^{n} \phi_{1 k}(-\tau)-a b \phi_{3 n}(0)
\end{array}\right) \quad \text { with } \phi \quad \begin{array}{c}
\phi_{11} \\
\phi_{12} \\
\vdots \\
\phi_{1 n} \\
\phi_{21} \\
\phi_{22} \\
\vdots \\
\phi_{2 n} \\
\phi_{31} \\
\phi_{32} \\
\vdots \\
\phi_{3 n}
\end{array}\right) \in \mathscr{C}
$$

Analogously, the linearisation of (2) about $E_{0}$ is expressible as

$$
\mathbf{u}^{\prime}(t)=L(a) \mathbf{u}_{t}
$$

where the linear operator $L(a): \mathscr{C} \rightarrow \mathbb{R}^{3 n}$ is defined as

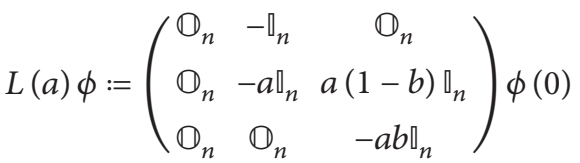

$$
\begin{aligned}
& +\left(\begin{array}{lll}
\mathbb{O}_{n} & \mathbb{O}_{n} & \mathbb{O}_{n} \\
\mathbb{P}_{n} & \mathbb{O}_{n} & \mathbb{O}_{n} \\
\mathbb{Q}_{n} & \mathbb{O}_{n} & \mathbb{O}_{n}
\end{array}\right) \phi(-\tau),
\end{aligned}
$$

$$
A(a) \phi=\phi^{\prime}, \quad \phi \in \operatorname{Dom}(A(a)), \operatorname{Dom}(A(a))=\left\{\phi \in \mathscr{C} \mid \phi^{\prime} \in \mathscr{C}, \phi^{\prime}(0)=L(a) \phi\right\},
$$

where $\mathbb{O}_{n}$ and $\mathbb{t}_{n}$ are the $n \times n$ zero and identity matrices, respectively. The $n \times n$ matrices $\mathbb{P}_{n}$ and $\mathbb{Q}_{n}$ are given by

$$
\begin{aligned}
& \mathbb{P}_{n}=\frac{1}{n}\left(\begin{array}{ccccc}
c+1 & 1 & 1 & \cdots & 1 \\
1 & c+1 & 1 & \cdots & 1 \\
\vdots & \vdots & \vdots & \ddots & \vdots \\
1 & 1 & 1 & \cdots & c+1
\end{array}\right) \\
& \mathbb{Q}_{n}=\frac{1}{n}\left(\begin{array}{ccccc}
1 & 1 & 1 & \cdots & 1 \\
1 & 1 & 1 & \cdots & 1 \\
\vdots & \vdots & \vdots & \ddots & \vdots \\
1 & 1 & 1 & \cdots & 1
\end{array}\right)
\end{aligned}
$$

It is well known that a linear functional differential equation such as (45) generates a strongly continuous semigroup of linear operators with infinitesimal generator $A(a)$ given by $[17,18]$ where the eigenvalues of $A(a)$ correspond to the roots of the characteristic equation (12).
Definition 3. Let $\mathbf{F}: \mathscr{C} \rightarrow \mathbb{R}^{3 n}$ and $\Gamma$ be a compact group. The system $\mathbf{x}^{\prime}(t)=\mathbf{F}\left(\mathbf{x}_{t}\right)$ is said to be $\Gamma$-equivariant if $\mathbf{F}\left(\gamma \mathbf{x}_{t}\right)=$ $\gamma \mathbf{F}\left(\mathbf{x}_{t}\right)$ for all $\gamma \in \Gamma$. 
Lemma 4. The nonlinear system (43) and the linear system (45) are $\mathbb{D}_{n^{n}}$-equivariant.

Proof. The proof is straightforward (see $[16,19,20])$.

\section{Equivariant Hopf Bifurcation}

The extension of the theory of equivariant Hopf bifurcation to functional differential equations was established in the series of papers $[18,21,22]$. Much of the development in this section is in the spirit of the work of $[16,19,20]$ that independently studied equivariant Hopf bifurcation in a ring of $n$ identical neurons characterised by signal transmission time delays. Defining the $3 n \times 1$ column vector

$$
\mathbf{u}(t)=\left[\begin{array}{llllllllllll}
y_{1}(t) & y_{2}(t) & \cdots & y_{n}(t) & x_{1}(t) & x_{2}(t) & \cdots & x_{n}(t) & w_{1}(t) & w_{2}(t) & \cdots & w_{n}(t)
\end{array}\right]^{\top},
$$

the linearisation (11) is expressible in the form

$$
\mathbf{u}^{\prime}(t)=\mathbf{A} \mathbf{u}(t)+\mathbf{B} \mathbf{u}(t-\tau)
$$

where $\mathbf{A}$ and $\mathbf{B}$ are $3 n \times 3 n$ matrices given by

$$
\begin{aligned}
& \mathbf{A}=\left(\begin{array}{ccc}
\mathbb{O}_{n} & -\mathbb{\Xi}_{n} & \mathbb{O}_{n} \\
\mathbb{O}_{n} & -a \mathbb{\Xi}_{n} & a(1-b) \mathbb{\square}_{n} \\
\mathbb{O}_{n} & \mathbb{O}_{n} & -a b \rrbracket_{n}
\end{array}\right), \\
& \mathbf{B}=\left(\begin{array}{lll}
\mathbb{O}_{n} & \mathbb{O}_{n} & \mathbb{O}_{n} \\
\mathbb{P}_{n} & \mathbb{O}_{n} & \mathbb{O}_{n} \\
\mathbb{Q}_{n} & \mathbb{O}_{n} & \mathbb{O}_{n}
\end{array}\right)
\end{aligned}
$$

From the above consideration, we get that the characteristic matrix of the linearisation of (2) about $E_{0}$ is given by the $3 n \times$ $3 n$ matrix

$$
L_{3 n}(\lambda):=\lambda \rrbracket_{3 n \times 3 n}-\mathbf{A}-\mathbf{B} e^{-\lambda \tau},
$$

and the associated characteristic equation is

$$
\operatorname{det} L_{3 n}(\lambda)=0 \text {, }
$$

which reduces to (12). Mitchell and Carr [7, 8] have shown that the characteristic matrix of the linearisation of (2) about the equilibrium $E_{0}$ is given by the $3 n \times 3 n$ block matrix of the form

$$
M_{3 n}(\lambda):=\left(\begin{array}{cccc}
D & E & E & \cdots \\
E & D & E & \cdots \\
E & E & D & \cdots \\
\vdots & \vdots & \vdots & \ddots
\end{array}\right)
$$

where $D$ and $E$ are given by

$$
\begin{aligned}
& D=\left(\begin{array}{ccc}
0 & -1 & 0 \\
\frac{(c+1)}{n} e^{-\lambda \tau} & -a & a(1-b) \\
\frac{e^{-\lambda \tau}}{n} & 0 & -a b
\end{array}\right), \\
& E=\left(\begin{array}{ccc}
0 & 0 & 0 \\
\frac{e^{-\lambda \tau}}{n} & 0 & 0 \\
\frac{e^{-\lambda \tau}}{n} & 0 & 0
\end{array}\right)
\end{aligned}
$$

It is evident that the characteristic matrix (54) is circulant, a fact to be exploited in the analysis to come. The corresponding characteristic equation is

$$
\operatorname{det} M_{3 n}(\lambda)=0
$$

which can be shown to reduce to (12). With the apparatus developed above, we are now in a position to establish some lemmas [16] that will lead us to an equivariant Hopf bifurcation theorem.

As a consequence of the fact that the matrix (54) is circulant and to facilitate the analysis to follow, we set $[16,18-$ 20]

$$
\begin{aligned}
& v_{j}:=\left(1, \chi^{j}, \chi^{2 j}, \ldots, \chi^{(3 n-1) j}\right)^{\top}, \\
& \quad j=0,1, \ldots, 3 n-1, \quad \chi=e^{2 i \pi / 3 n} .
\end{aligned}
$$

We note the following identities:

$$
\begin{gathered}
\chi^{3 n j}=1, \\
\chi^{(3 n-1) j}=\chi^{-j}, \\
\chi^{(3 n-2) j}=\chi^{-2 j}, \\
\vdots \\
\chi^{(3 n-k) j}=\chi^{-k j} .
\end{gathered}
$$


We arrive at the following useful results:

$$
\begin{aligned}
& \mathbf{B} v_{j}=\frac{1}{n}\left(\begin{array}{c}
0 \\
0 \\
\vdots \\
0 \\
1+(c+1) \chi^{j}+\chi^{2 j}+\cdots+\chi^{(3 n-1) j} \\
\vdots \\
1+\chi^{j}+\chi^{2 j}+\cdots+(c+1) \chi^{(3 n-1) j} \\
1+\chi^{j}+\chi^{2 j}+\cdots+\chi^{(3 n-1) j} \\
1+\chi^{j}+\chi^{2 j}+\cdots+\chi^{(3 n-1) j} \\
\vdots \\
1+\chi^{j}+\chi^{2 j}+\cdots+\chi^{(3 n-1) j}
\end{array}\right) \\
& \left(\begin{array}{c}
0 \\
0 \\
\vdots \\
0 \\
c+v_{0}^{\mathrm{T}} v_{j} \\
c \chi^{j}+v_{0}^{\mathrm{T}} v_{j} \\
\vdots \\
c \chi^{-j}+v_{0}^{\mathrm{T}} v_{j} \\
v_{0}^{\mathrm{T}} v_{j} \\
v_{0}^{\mathrm{T}} v_{j} \\
\vdots \\
v_{0}^{\mathrm{T}} v_{j}
\end{array}\right), \quad v_{0}^{\mathrm{T}} v_{j}=\chi^{j}+\chi^{-j}+3 n-1 \text {, } \\
& v_{i}^{\top} \cdot \bar{v}_{j}=3 n, \\
& v_{j}=\bar{v}_{3 n-j}, \quad(j=0,1,2, \ldots, 3 n-1) .
\end{aligned}
$$

Following the footsteps of Lemma 3.1 of [16], we arrive at the following result.

Lemma 5. Eigenvalue conditions are formulated as follows. Let $c, n$, and $\tau$ be fixed such that there is a solution, $\left(\omega_{c}, a_{c}\left(\omega_{c}\right)\right)$, of (17). Then,

(A1) the characteristic matrix $M_{3 n}(\lambda)$ is continuously differentiable with respect to $a$;

(A2) the infinitesimal generator, $A(a)$, of the linear operator (46) has a multiplicity $(n-1)$ pair of eigenvalues, $\pm i \omega_{c}$, at $a=a_{c}$;
(A3) the generalised eigenspace, $P_{ \pm i \omega_{c}}\left(A\left(a_{c}\right)\right)$, of $A\left(a_{c}\right)$ for $\pm i \omega_{c}$ is spanned by the $2(n-1)$ eigenvectors $\mathbf{v}$ implicitly defined by the relationship

$$
\left[A_{3 n \times 3 n}\left(a_{c}\right)-\lambda \cdot \rrbracket_{3 n \times 3 n}\right]^{\ell} \mathbf{v}=\mathbf{0}, \quad \ell=1,2, \ldots, n-1,
$$

where $\lambda$ is a multiplicity $(n-1)$ eigenvalue of $A\left(a_{c}\right)$.

Proof. The differentiability of the Jacobian matrix $M_{3 n}(\lambda)$ follows from its definition in (54). Let us note first that characteristic equation (12) may be expressed in the format

$$
D(\lambda, \tau):=\Delta_{1}^{n-1}(\lambda, \tau) \cdot \Delta_{2}(\lambda, \tau)=0 .
$$

As a consequence of the symmetry imposed by the global coupling as depicted in Figure 1, it follows that the determinant $M_{3 n}-\lambda \cdot \rrbracket_{3 n \times 3 n}$ is factorisable, and there are multiplicity $(n-1)$ roots [7]. From the discussion in Section 1 and also under the conditions of the lemma, it is evident that $\Delta_{j}\left( \pm i \omega_{c}, \tau\right)=0$. This means that the characteristic equation (12) indeed admits a multiplicity $(n-1)$ pair of roots, $\pm i \omega_{c}$. Consequently, (44) implies that $A\left(a_{c}\right)$ has a multiplicity $(n-1)$ pair of complex conjugate eigenvalues $\pm i \omega_{c}$. Employing the properties of $v_{j}$, we can show that

$$
\begin{gathered}
M_{3 n}(\lambda) v_{j}=\Delta_{j}(\lambda, \tau) v_{j}, \\
M_{3 n-j}(\lambda) v_{j}=\Delta_{j}(\lambda, \tau) v_{3 n-j} .
\end{gathered}
$$

Hence the conditions of the lemma lead to

$$
\begin{aligned}
M_{3 n}\left(i \omega_{c}\right) v_{j} & =M_{3 n}\left(i \omega_{c}\right) v_{3 n-j}=M_{3 n}\left(-i \omega_{c}\right) \bar{v}_{j} \\
& =M_{3 n}\left(-i \omega_{c}\right) \bar{v}_{3 n-j}=0 .
\end{aligned}
$$

Noting first that $v_{3 n-j}=\bar{v}_{j}$ and then using a standard result from [17], we arrive at the conclusion that the eigenvectors of $A\left(a_{c}\right)$ corresponding to $\pm i \omega_{c}$ are given by (19) to (21) in [7]. The generalised eigenspace $P_{ \pm i \omega_{c}}\left(A\left(a_{c}\right)\right)$ is spanned by these $2(n-1)$ linearly independent eigenvectors corresponding to the $2(n-1)$ eigenvalues [16]. An alternative approach to proving (A3) is given on page 24 of [19].

In the characteristic equation (12), let $\lambda=\lambda(\tau)$ and differentiate with respect to $\tau$ to obtain

$$
\begin{aligned}
& {\left[3 \lambda^{2}+2 a(1+b) \lambda+a^{2} b\right.} \\
& -\tau\left[\lambda\left(1+\frac{c}{n}\right)+a\left(1+\frac{b c}{n}\right)\right] e^{-\lambda \tau}+\left(1+\frac{c}{n}\right) \\
& \cdot e^{-\lambda \tau}+(n-1) F_{s}(\lambda, \tau) \\
& \left.\cdot\left(F_{a p}(\lambda, \tau)+\left(2 \lambda+a-\frac{c \tau}{n} e^{-\lambda \tau}\right) F_{1}(\lambda)\right)\right] \frac{d \lambda}{d \tau} \\
& =\lambda\left[\lambda\left(1+\frac{c}{n}\right)+a\left(1+\frac{b c}{n}\right)\right]+\frac{c \lambda(n-1)}{n} \\
& \cdot F_{1}(\lambda) F_{s}(\lambda, \tau),
\end{aligned}
$$


from which it is straightforward to show that the usual transversality condition

$$
\operatorname{Re}\left(\left.\frac{d \lambda}{d \tau}\right|_{\lambda=i \omega}\right) \neq 0
$$

is fulfilled if, and only if,

$$
Q_{1} Q_{2} \neq 0
$$

where

$$
\begin{aligned}
Q_{1}:= & a^{3} b n^{2}-n a^{3} b+a b \omega^{2} n^{2}-2 \omega^{2} c a b-a b n \omega^{2} \\
& +2 \omega^{2} c a b n+2 \omega^{2} c a n-2 \omega^{2} c a-c-n
\end{aligned}
$$

and where relations (18) have been used to perform some simplifications in (66). The expression for $\mathbb{Q}_{2}$ is given in Appendix. Once again, following the footsteps of Lemma 3.2 of [16], we arrive at the following nonresonance condition.

Lemma 6. The nonresonance condition is formulated as follows. Let $b, c, n$, and $\tau$ be fixed such that there exists a solution $\left(\omega_{c}, a_{c}\left(\omega_{c}\right)\right)$ of (36). If $b, c, n, \tau$, and $a_{c}$ are such that, for each $m=2,3, \ldots$, at least one of the following holds,

$$
\begin{aligned}
& a_{c} b {\left[-n m \omega_{c}^{2}+c \cdot \cos \left(m \omega_{c} \tau\right)\right] } \\
&-m \omega_{c}\left[a_{c} n m \omega_{c}-c \cdot \sin \left(m \omega_{c} \tau\right)\right] \neq 0, \\
& a_{c} b\left[n m a_{c} \omega_{c}-c \cdot \sin \left(m \omega_{c} \tau\right)\right] \\
&-m \omega_{c}\left[-n m^{2} \omega_{c}^{2}+c \cdot \cos \left(m \omega_{c} \tau\right)\right] \neq 0,
\end{aligned}
$$

then all other eigenvalues of $A\left(a_{c}\right)$ are not integer multiples of $\pm i \omega_{c}$.

Proof. It is evident from (12) and (16) that, for $a=a_{c}$, $\Delta_{j}\left( \pm i \omega_{c}, \tau\right) \neq 0$. This implies that one-to-one resonances cannot happen. However, other resonances are feasible and do occur if there exists $\mathbb{Z} \ni m>1$ such that (16) are fulfilled for $\omega=\omega_{c}$ and $\omega=m \omega_{c}$, with identical values of the parameters $a, b, c, n, \tau$ [16]. This is precluded from happening by (68) [16].

We thus arrive at the following result.

Theorem 7. Let $b, c, n$, and $\tau$ be fixed such that there exists a solution $\left(\omega_{c}, a_{c}\left(\omega_{c}\right)\right)$ of (36). If conditions (66) and (68) are satisfied, then system (2) undergoes an equivariant Hopf bifurcation on the parametric surfaces given by (16) as the parameter a varies through $a_{c}$.

\section{Appendix}

The quantity $\mathbb{Q}_{2}$ appearing in (66) is given by the lengthy expression

$$
\begin{aligned}
Q_{2} & =\left(4 a^{4} n^{4} \omega^{6} \tau b^{3}+2 a^{6} n^{4} \omega^{4} \tau b^{4}+4 a^{6} n^{4} \omega^{4} \tau b^{3}\right. \\
& -2 a^{3} c^{2} n b^{2} \omega^{2}+4 \omega^{2} n^{2} c a^{4} b^{4}+6 \omega^{4} n^{2} c a^{2} b^{2} \\
& +2 a^{3} c^{2} n^{2} b^{2} \omega^{2}-a^{2} \tau \omega^{6} n^{2} c-\omega^{2} c a^{7} b^{6} n^{2}
\end{aligned}
$$

$$
\begin{aligned}
& +5 \omega^{4} c a^{5} b^{5} n^{2}+a^{2} \tau \omega^{6} n^{3} c+a^{7} b^{6} n^{3} \omega^{2} c \\
& -5 a^{5} b^{5} n^{3} \omega^{4} c-n^{2} \tau \omega^{6} a c+2 a b n^{2} \omega^{6} c \\
& +4 a^{5} b^{5} n^{2} \omega^{2} c-2 a b n^{3} \omega^{6} c-4 a^{5} b^{5} n^{3} \omega^{2} c \\
& -2 \omega^{6} n^{3} \tau a^{4} b^{4}+2 \omega^{6} n^{4} \tau a^{4} b^{4}-6 a^{3} b^{3} n^{3} \omega^{4} c \\
& -a^{6} \tau b^{5} c^{2} n^{2}-a^{6} b^{6} c^{3} \tau n+a^{6} \tau b^{5} c^{2} n \\
& +6 a^{3} b^{3} n^{2} \omega^{4} c-2 \tau \omega^{4} n^{3} a^{3} b^{3}-2 \tau \omega^{6} n^{3} a b \\
& +4 a^{4} n^{4} \omega^{6} \tau b-4 a^{4} n^{3} \omega^{6} \tau b-2 \tau \omega^{2} n^{3} a^{5} b^{3} \\
& -2 \tau \omega^{4} n^{3} a^{3} b+a^{6} b^{6} \tau n^{3} \omega^{2} c+2 a^{4} b^{2} \tau \omega^{4} n^{3} c \\
& +2 a^{6} b^{6} n^{3} \omega^{4} \tau c-2 a^{4} b^{2} \tau \omega^{4} n^{2} c-n^{2} \tau a^{5} b^{5} \omega^{2} c \\
& -2 n^{2} \tau a^{3} b^{2} \omega^{4} c-4 n^{3} a^{5} \omega^{2} c b^{4}-7 n^{3} a^{3} \omega^{4} c b^{2} \\
& -n^{3} a^{3} b c \omega^{4}-4 n^{3} \omega^{4} a^{5} c b^{4}-7 n^{3} \omega^{6} a^{3} c b^{2} \\
& +4 \omega^{4} c a^{5} n^{2} b^{4}+7 \omega^{6} c a^{3} n^{2} b^{2}+4 a^{5} \omega^{2} n^{2} c b^{4} \\
& +7 a^{3} \omega^{4} n^{2} c b^{2}+a^{3} b n^{2} c \omega^{4}+a^{4} b^{4} c^{3} \tau \omega^{2} \\
& -a^{2} b^{2} c^{3} \tau \omega^{4}+2 a^{3} b^{3} c^{3} n \omega^{2}+a b c^{3} n \omega^{4} \\
& +a^{2} b n^{2} c \omega^{4}+2 \omega^{4} c a^{5} n^{2} b^{3}+2 \omega^{6} c a^{3} n^{2} b \\
& -2 n^{3} \omega^{4} a^{5} c b^{3}-2 n^{3} \omega^{6} a^{3} c b-8 n^{3} \omega^{6} a^{3} c b^{3} \\
& -3 n^{3} \omega^{8} a c b+8 \omega^{6} c a^{3} n^{2} b^{3}+3 \omega^{8} c a n^{2} b \\
& -4 a^{2} n^{3} \omega^{8} \tau b-4 a^{4} n^{3} \omega^{6} \tau b^{3}-2 a^{6} n^{3} \omega^{4} \tau b^{4} \\
& -4 a^{6} n^{3} \omega^{4} \tau b^{3}+4 a^{2} n^{4} \omega^{8} \tau b+2 n^{3} a^{7} b^{5} \omega^{2} \\
& -2 a^{2} n^{4} \omega^{8} \tau+2 a^{2} n^{3} \omega^{8} \tau-2 n^{4} \omega^{4} a^{5} b^{5} \\
& +2 n^{3} \omega^{4} a^{5} b^{5}-6 n^{4} \omega^{6} a^{3} b^{2}-6 n^{4} \omega^{4} a^{5} b^{2} \\
& -2 n^{4} \omega^{2} a^{7} b^{5}+n^{3} \omega^{2} a^{4} b^{4}+2 n^{3} \omega^{2} a^{4} b^{3} \\
& +2 n^{3} \omega^{4} a^{2} b-2 a^{3} b^{3} c^{3} \omega^{2}-a b c^{3} \omega^{4}-a^{5} c^{2} n b^{4} \\
& -a c^{2} n \omega^{4}+a^{5} c^{2} n^{2} b^{4}+a c^{2} n^{2} \omega^{4}-10 n^{4} \omega^{6} a^{3} b^{3} \\
& +10 n^{3} \omega^{6} a^{3} b^{3}-10 n^{4} \omega^{4} a^{5} b^{3}+6 n^{3} \omega^{6} a^{3} b^{2} \\
& +10 n^{3} \omega^{4} a^{5} b^{3}+6 n^{3} \omega^{4} a^{5} b^{2}+\omega^{6} c^{3} \tau n-2 n^{3} a \omega^{6} c \\
& +n^{3} a^{7} b^{5} c+n^{3} a^{7} b^{6} c-2 n^{3} \omega^{8} a c+2 \omega^{8} c a n^{2} \\
& +2 a \omega^{6} n^{2} c-a^{7} b^{5} n^{2} c-a^{7} b^{6} n^{2} c+a^{6} b^{6} c^{3} \tau \\
& +\omega^{6} \tau c^{2} n^{2}-\omega^{6} c^{2} \tau n+a^{5} b^{5} c^{3} n-a^{6} b^{5} n^{2} c \\
& +\omega^{10} n^{2} \tau c-\omega^{10} n^{3} \tau c-2 a^{4} \tau b^{3} c^{2} n^{2} \omega^{2} \\
& -a^{2} \tau b c^{2} n^{2} \omega^{4}+2 a^{4} \tau b^{3} c^{2} n \omega^{2}+a^{2} \tau b c^{2} n \omega^{4}
\end{aligned}
$$




$$
\begin{aligned}
& -2 a^{6} b^{5} n^{2} \omega^{4} \tau c-2 a^{6} b^{4} n^{2} \omega^{4} \tau c+2 \omega^{4} n^{3} a^{6} \tau b^{5} c \\
& +2 \omega^{4} n^{3} a^{6} \tau b^{4} c+a^{8} b^{6} \omega^{2} \tau n^{3} c+2 a^{6} b^{5} \tau \omega^{2} n^{3} c \\
& +2 a^{4} b^{4} \tau \omega^{4} n^{3} c+a^{2} b^{2} \tau \omega^{6} n^{3} c-a^{8} b^{6} \omega^{2} \tau n^{2} c \\
& -2 a^{6} b^{5} \tau \omega^{2} n^{2} c-2 a^{4} b^{4} \tau \omega^{4} n^{2} c-a^{2} b^{2} \tau \omega^{6} n^{2} c \\
& -a^{6} b^{6} \tau n^{2} \omega^{2} c+2 \omega^{10} n^{3} \tau-2 \omega^{10} n^{4} \tau+2 n^{4} \omega^{8} a \\
& +2 n^{4} a^{3} \omega^{6}-a^{5} b^{5} c^{3}-\omega^{6} c^{3} \tau+2 \omega^{6} n^{2} c-2 a^{3} n^{3} \omega^{6} \\
& -2 n^{3} \omega^{8} a-2 a^{6} b^{6} n^{2} \omega^{4} \tau c-n^{3} \omega^{6}-a^{6} \tau \omega^{2} n^{2} c b^{4} \\
& -4 a^{4} \tau \omega^{4} n^{2} c b^{3}+2 \omega^{10} n^{3} \tau-2 \omega^{10} n^{4} \tau+2 n^{4} \omega^{8} a \\
& +2 n^{4} a^{3} \omega^{6}-a^{5} b^{5} c^{3}-\omega^{6} c^{3} \tau+2 \omega^{6} n^{2} c-2 a^{3} n^{3} \omega^{6} \\
& -2 n^{3} \omega^{8} a-2 a^{6} b^{6} n^{2} \omega^{4} \tau c-n^{3} \omega^{6}-a^{6} \tau \omega^{2} n^{2} c b^{4} \\
& -4 a^{4} \tau \omega^{4} n^{2} c b^{3}-2 a^{2} \tau \omega^{6} n^{2} c b+a^{6} \tau \omega^{2} n^{3} c b^{4} \\
& +4 a^{4} \tau \omega^{4} n^{3} c b^{3}+2 a^{2} \tau \omega^{6} n^{3} c b-n^{2} \tau \omega^{2} a^{5} c b^{4} \\
& -2 n^{2} \tau \omega^{4} a^{3} c b^{3}-n^{2} \tau \omega^{6} a c b-\omega^{2} c^{3} \tau n a^{4} b^{4} \\
& +\omega^{4} c^{3} \tau n a^{2} b^{2}+\omega^{2} \tau c^{2} n^{2} a^{4} b^{4}+2 \omega^{4} \tau c^{2} n^{2} a^{2} b^{2} \\
& -\omega^{2} c^{2} \tau n a^{4} b^{4}-2 \omega^{4} c^{2} \tau n a^{2} b^{2}-2 \omega^{8} n^{2} \tau c a^{2} b \\
& -3 \omega^{6} n^{2} \tau c a^{4} b^{4}-4 \omega^{6} n^{2} \tau c a^{4} b^{3}-\omega^{6} n^{2} \tau c a^{4} b^{2} \\
& +2 \omega^{8} n^{3} \tau c a^{2} b+3 \omega^{6} n^{3} \tau c a^{4} b^{4}+4 \omega^{6} n^{3} \tau c a^{4} b^{3} \\
& \left.+\omega^{6} n^{3} \tau c a^{4} b^{2}\right) \text {. }
\end{aligned}
$$

The coefficients of the octic polynomial (22) are given by the following:

$$
\begin{aligned}
A_{8}= & n^{4}+2 n^{3} c+n^{2} c^{2} \\
A_{6}= & -n^{4} a^{2}-4 a^{2} n^{2} c^{2} b-6 n^{3} a^{2} b c-2 a^{2} n^{3} c \\
& -2 n^{3} a^{2} b^{2} c-n^{2} a^{2} b^{2} c^{2}-4 n^{4} a^{2} b \\
A_{5}= & -4 n^{3} a^{3} b^{2} c-4 a^{3} n^{3} b c-2 a^{3} n^{4}-2 n^{4} a^{3} b \\
& -2 a^{3} n^{2} b^{2} c^{2}-2 n^{2} a^{3} b^{3} c^{2}, \\
A_{4}= & 5 a^{4} n^{2} b^{2} c^{2}+2 n^{2} a^{4} b^{4} c^{2}+4 n^{2} a^{4} b^{3} c^{2} \\
& +6 n^{3} a^{4} b^{3} c+4 n^{4} a^{4} b+5 n^{4} a^{4} b^{2} \\
& +10 n^{3} a^{4} b^{2} c+6 a^{4} n^{3} b c+2 a^{4} n^{4} \\
A_{3}= & 2 n^{2} a^{5} b^{4} c^{2}+4 n^{3} a^{5} b^{3} c+2 n^{2} a^{5} b^{3} c^{2}+2 n^{4} a^{5} b^{2} \\
& +4 n^{3} a^{5} b^{2} c+2 n^{4} a^{5} b,
\end{aligned}
$$

$$
\begin{aligned}
A_{2}= & -n^{2} a^{2} b^{2} c^{2}-4 a^{2} n^{2} c^{2} b-2 a^{2} n c^{3} b^{2}+2 n^{3} a^{6} b^{3} c \\
& -a^{2} c^{2} n^{2}-2 a^{2} n^{3} c-a^{2} c^{4} b^{2}-n^{4} a^{2} \\
& -2 n^{3} a^{2} b c+n^{4} a^{6} b^{2}-2 a^{2} c^{3} n b+n^{2} a^{6} b^{4} c^{2}, \\
A_{1}= & 2 a^{3} n^{4}+6 a^{3} n^{2} b^{2} c^{2}+2 a^{3} c n^{3}+2 a^{3} c^{4} b^{3} \\
& +6 a^{3} n^{3} b c+2 a^{3} n b^{3} c^{3}+6 a^{3} c^{2} n^{2} b \\
& +6 a^{3} c^{3} n b^{2}, \\
A_{0}= & -4 a^{4} n^{3} b c-6 a^{4} n^{2} b^{2} c^{2}-4 a^{4} n b^{3} c^{3}-a^{4} c^{4} b^{4} \\
& -a^{4} n^{4} .
\end{aligned}
$$

\section{Conflict of Interests}

The author declares that there is no conflict of interests regarding the publication of this paper.

\section{References}

[1] M. Recker, S. Nee, P. C. Bull et al., "Transient cross-reactive immune responses can orchestrate antigenic variation in malaria," Nature, vol. 429, no. 6991, pp. 555-558, 2004.

[2] K. B. Blyuss and S. Gupta, "Stability and bifurcations in a model of antigenic variation in malaria," Journal of Mathematical Biology, vol. 58, no. 6, pp. 923-937, 2009.

[3] K. B. Blyuss, "The effects of symmetry on the dynamics of antigenic variation," Journal of Mathematical Biology, vol. 66, no. 1-2, pp. 115-137, 2013.

[4] K. B. Blyuss and Y. N. Kyrychko, "Symmetry-breaking in a model of antigenic variation with immune delay," Bulletin of Mathematical Biology, vol. 74, no. 10, pp. 2488-2509, 2012.

[5] B. S. Chan and P. Yu, "Synchrony-breaking Hopf bifurcation in a model of antigenic variation," International Journal of Bifurcation and Chaos in Applied Sciences and Engineering, vol. 23, no. 2, Article ID 1350021, 2013.

[6] I. Ncube, "Absolute stability and Hopf bifurcation in a Plasmodium falciparum malaria model incorporating discrete immune response delay," Mathematical Biosciences, vol. 243, no. 1, pp. 131-135, 2013.

[7] J. L. Mitchell and T. W. Carr, "Synchronous versus asynchronous oscillations for antigenically varying Plasmodium falciparum with host immune response," Journal of Biological Dynamics, vol. 6, no. 2, pp. 333-357, 2012.

[8] J. L. Mitchell and T. W. Carr, "Oscillations in an intra-host model of Plasmodium Falciparum malaria due to cross-reactive immune response," Bulletin of Mathematical Biology, vol. 72, no. 3, pp. 590-610, 2010.

[9] F. E. McKenzie and W. H. Bossert, "The dynamics of Plasmodium falciparum blood-stage infection," Journal of Theoretical Biology, vol. 188, no. 1, pp. 127-140, 1997.

[10] F. Ellis McKenzie and W. H. Bossert, "A target for intervention in Plasmodium falciparum infections," The American Journal of Tropical Medicine and Hygiene, vol. 58, no. 6, pp. 763-767, 1998.

[11] P. De Leenheer and S. S. Pilyugin, "Immune response to a malaria infection: properties of a mathematical model," Journal of Biological Dynamics, vol. 2, no. 2, pp. 102-120, 2008. 
[12] M. Golubitsky and D. G. Schaeffer, Singularities and Groups in Bifurcation Theory, Volume II. Applied Mathematical Sciences, vol. 69, Springer, New York, NY, USA, 1988.

[13] M. Golubitsky and I. Stewart, The Symmetry Perspective: From Equilibrium to Chaos in Phase Space and Physical Space, vol. 200 of Progress in Mathematics, Birkhäuser, Basel, Switzerland, 2002.

[14] M. Golubitsky, I. Stewart, and A. Török, "Patterns of synchrony in coupled cell networks with multiple arrows," SIAM Journal on Applied Dynamical Systems, vol. 4, no. 1, pp. 78-100, 2005.

[15] M. Golubitsky and I. Stewart, "Nonlinear dynamics of networks: the groupoid formalism," Bulletin of the American Mathematical Society, vol. 43, no. 3, pp. 305-364, 2006.

[16] S. A. Campbell, Y. Yuan, and S. D. Bungay, "Equivariant Hopf bifurcation in a ring of identical cells with delayed coupling," Nonlinearity, vol. 18, no. 6, pp. 2827-2846, 2005.

[17] J. K. Hale and S. M. V. Lunel, Introduction to Functional Differential Equations, Springer, New York, NY, USA, 1993.

[18] J. Wu, "Symmetric functional-differential equations and neural networks with memory," Transactions of the American Mathematical Society, vol. 350, no. 12, pp. 4799-4838, 1998.

[19] S. Guo and L. Huang, "Hopf bifurcating periodic orbits in a ring of neurons with delays," Physica D: Nonlinear Phenomena, vol. 183, no. 1-2, pp. 19-44, 2003.

[20] D. Fan and J. Wei, "Equivariant Hopf bifurcation in a ring of identical cells with delay," Mathematical Problems in Engineering, vol. 2009, Article ID 950251, 34 pages, 2009.

[21] W. Krawcewicz, P. Vivi, and J. Wu, "Computation formulae of an equivariant degree with applications to symmetric bifurcations," Nonlinear Studies, vol. 4, no. 1, pp. 89-119, 1997.

[22] W. Krawcewicz and J. Wu, "Theory and applications of Hopf bifurcations in symmetric functional differential equations," Nonlinear Analysis: Theory, Methods \& Applications, vol. 35, no. 7, pp. 845-870, 1999. 


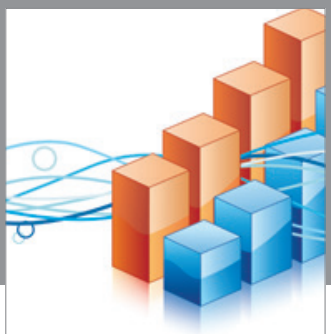

Advances in

Operations Research

vatem alat4

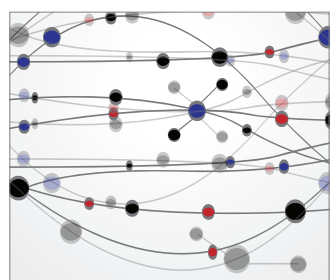

\section{The Scientific} World Journal
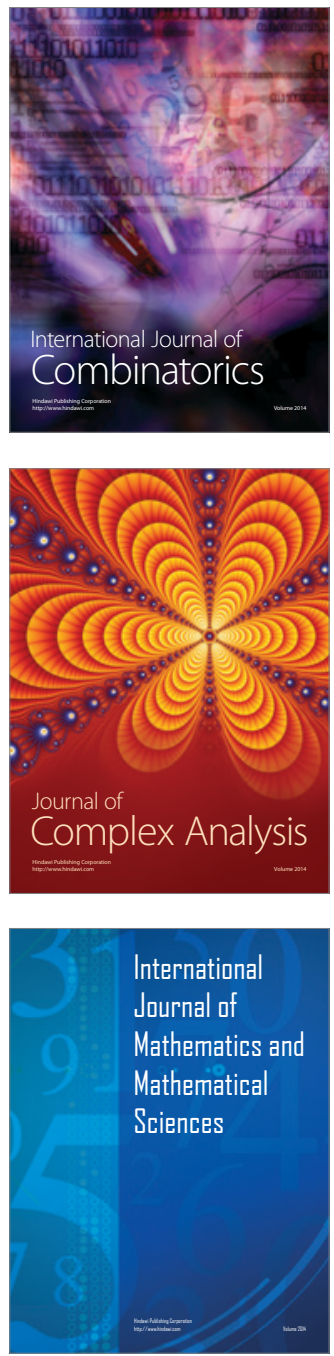
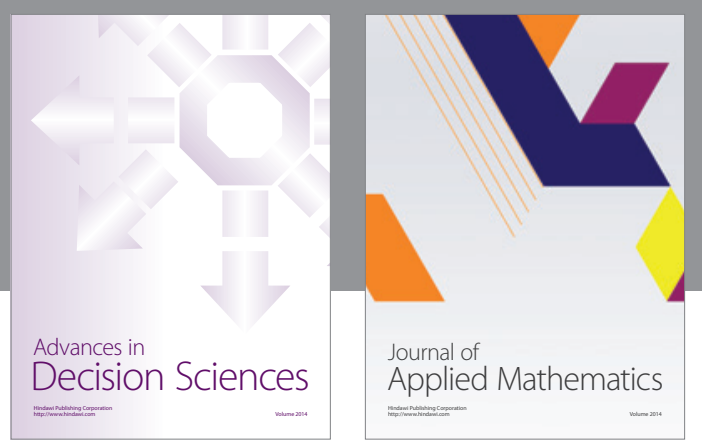

Algebra

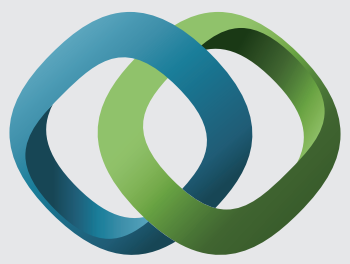

\section{Hindawi}

Submit your manuscripts at

http://www.hindawi.com
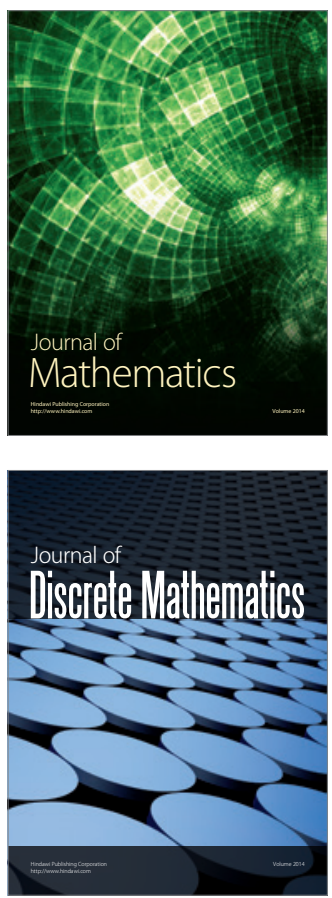

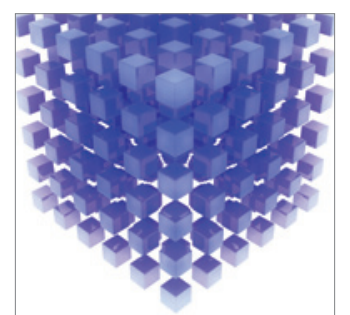

Mathematical Problems in Engineering
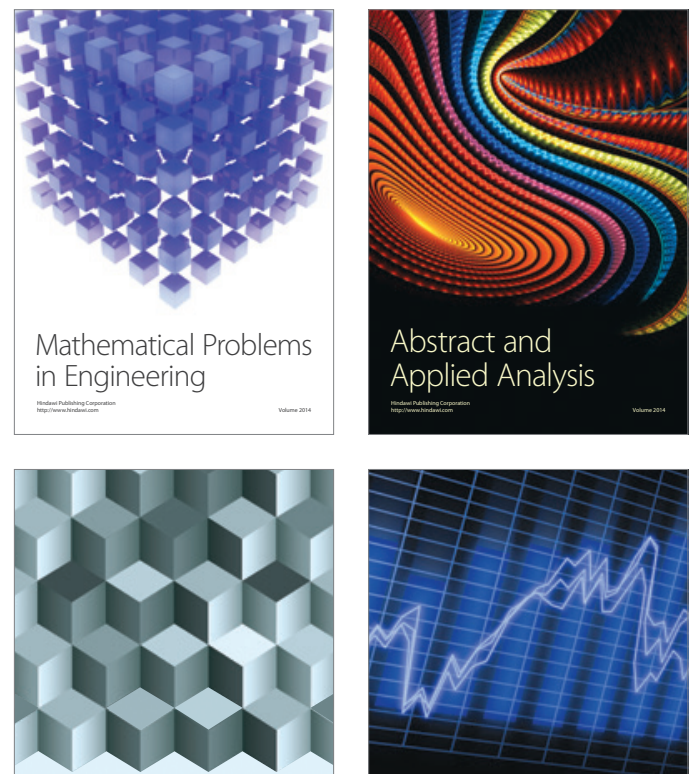

Journal of

Function Spaces

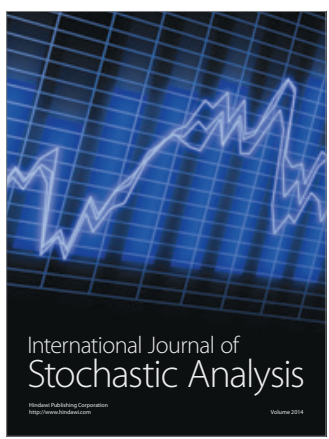

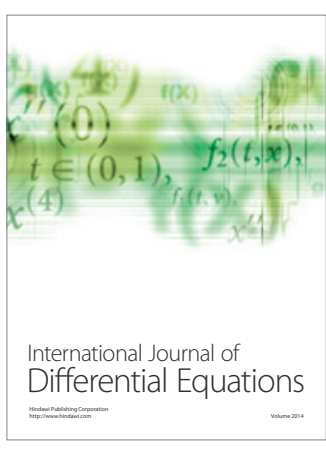
